\title{
Dressler's syndrome after right ventricular infarction
}

\author{
JONATHAN STREIFLER \\ M.D. \\ SHLOMO DUX \\ M.D. \\ Chaim HellmaN* \\ M.D.
}

\author{
SilVio PITLIK \\ M.D. \\ GERSHON PERRY \\ M.D. \\ MARK GREENWALD \\ M.D.
}

\author{
JOSEPH B. ROSENFELD \\ M.D.
}

Department of Internal Medicine ' $C$ ' and *the Massada Unit of Nuclear Cardiology, Beilinson Medical Center, Petah Tiqva and Tel Aviv University Sackler School of Medicine, Israel

\section{Summary}

Undiagnosed myocardial infarction of the right ventricle presented as Dressler's syndrome. Radioisotopic diagnostic procedures including first-pass and multigated acquisition nuclear angiography (MUGA) and thallium-201 perfusion studies enabled a retroactive diagnosis of myocardial infarction, showed it to be in the right ventricle, and clarified the aetiology of the unexplained fever and pleuropericarditis.

KEY WORDS: aortic incompetence, pleuropericarditis.

A diagnosis of Dressler's syndrome requires evidence of myocardial infarction, followed after a variable interval of time, by fever, pericarditis and pleuritis (Dressler, 1959; Dressler and Leavitt, 1960). Classically, the myocardial necrosis is located in the left ventricle and the diagnosis of myocardial infarction has already been established when pleuropericarditis and fever appear (Dressler, 1959; Dressler and Leavitt, 1960).

We report a patient with a right ventricular myocardial infarction (RVMI) complicated by Dressler's syndrome. RVMI, as is often the case, had remained undiagnosed. Unexplained fever and pleuropericarditis prompted the retroactive isotope proof of RVMI and confirmed the proposed diagnosis of Dressler's syndrome.

Reprint requests: Professor J. Rosenfeld, Department of Internal Medicine ' $C$ ' and The Renal Unit, Beilinson Medical Center, Petah Tiqva, 49 100, Israel.

\section{Case report}

A 62-year-old Caucasian male was admitted for investigation of fever of 6-weeks duration. Eighteenoo years previously, he had been hospitalized because of acute myocardial ischaemia of the anterior walkôto the left ventricle. During follow-up, hypertension.o $180 / 100 \mathrm{mmHg}$ was treated with chlorthalidone $50 \mathrm{D}$ $\mathrm{mg}$ every other day. Because of persistent angina占 syndrome 5 years before the present admission, heo underwent coronary arteriography which revealed $a \overrightarrow{\overrightarrow{0}}$ $50 \%$ occlusion of the left anterior descending coro-3 nary artery, and irregularity of the intima of the righe coronary artery. Regional wall motion was normal in all aspects and the left ventricle global ejections fraction was $72 \%$ (lower limit for our laboratory is 55\%). Since that time he was known to have a stable anginal syndrome which caused chest pain uponmoderate physical exertion. He was also known too have mild aortic valve insufficiency. Three months before admission, he had angina occurring at rest ando fever of $\mathbf{2}$ days duration without leukocytosis. Isoen- $\rightarrow$ zyme studies for evidence of myocardial necrosis? were within normal limits. Electrocardiogram (ECG) showed a transient $\mathrm{T}$-wave inversion in the III and ${ }^{\mathrm{O}}$ AVF frontal plane leads. As no clear evidence was 0 found for myocardial infarction and the fever sub N sided spontaneously, he was sent home.

Six weeks later the patient developed fever reach ing $38.0^{\circ} \mathrm{C}$ accompanied by weakness and anorexia. He was treated with co-trimoxazole and ampicillines without clinical improvement. During the week before admission, the patient noted moderate pain-o 
less swelling of the lower legs. Physical examination revealed blood pressure $130 / 90 \mathrm{mmHg}$, and temperature $37 \cdot 8^{\circ} \mathrm{C}$. There were no signs of venous congestion in the neck whereas the liver was tender and there was pitting oedema of both lower limbs. ECG showed a sinus rhythm and negative T-waves in II, III and AVF. No pathological Q-waves or signs of pericarditis were present.

On the third day of hospitalization, a pericardial friction rub was detected over the left parasternal area.

Laboratory studies showed ESR (Westergren) 30 $\mathrm{mm}$ in the first hour, WBC $8 \times 10^{9} / 1$ with a normal differential. Biochemistry, autoimmune serology and blood cultures were unrevealing. Chest X-ray showed mild enlargement of the heart shadow by the left ventricle. A pleural effusion was seen in the left costophrenic angle.

An echocardiography (M-mode) study showed a mild pericardial effusion estimated at $150 \mathrm{ml}$, an increased end diastolic dimension of the right ventricle, a slight delay in the movement of the septum and hypokinesia of the free wall of the right ventricle.

On admission, diuretic therapy with frusemide 40 $\mathrm{mg} /$ day was begun. Seven days later, when the diuretic was stopped, there was significant venous congestion of neck veins and an increasing oedema of the legs. No signs of left heart failure were found on physical examination and repeated chest X-rays.

Multigated acquisition nuclear angiography (MUGA) was performed 5 days after admission and again 5 weeks later. The results in both examinations and in all views showed the right ventricular ejection fraction to be significantly far below normal (18\%: normal values in our laboratory are $52 \pm 7 \%$ ).

Akinesia of the free wall and hypokinesia of the inferior wall were clearly demonstrated. The left ventricle, on the other hand, appeared in all examinations and views to be normal at rest, while on effort the ejection fraction dropped to $46 \%$ (the lower limit of normal is $55 \%$ ), and hypokinesia of the inferior wall was demonstrated.

Fig. 1 illustrates the perimeter of both ventricles at end systole and end diastole. The perimetry of the right ventricle in systole remains almost unchanged from that in diastole. Thallium-201 perfusion studies showed normal accumulation of radioactive material throughout the left ventricle in contrast to the complete absence of perfusion of the right ventricle in all views under all conditions. Fig. 2 shows a left anterior oblique view of the heart during rest. The lack of right ventricular uptake in this figure is typical of the results obtained.

Treatment was initiated with aspirin $-4 \mathrm{~g}$ daily. After 2 days of this regimen, the general condition of the patient improved, fever returned to normal, the pleural effusion resolved and the pericardial friction

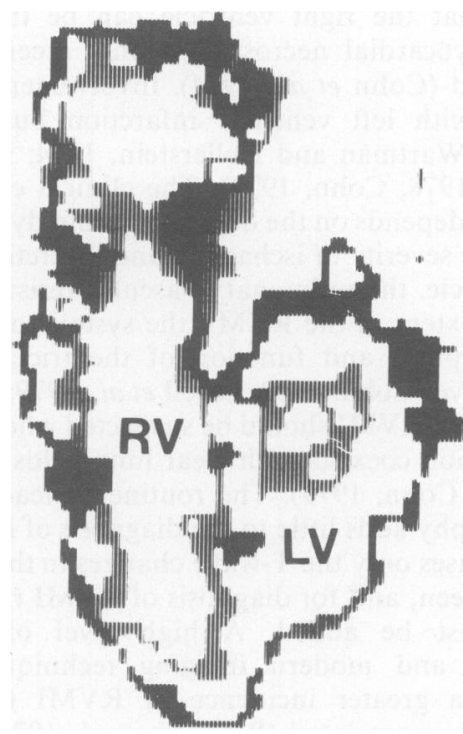

FIG. 1. Perimeter of ventricles at end systole (light) and end diastole (dark).

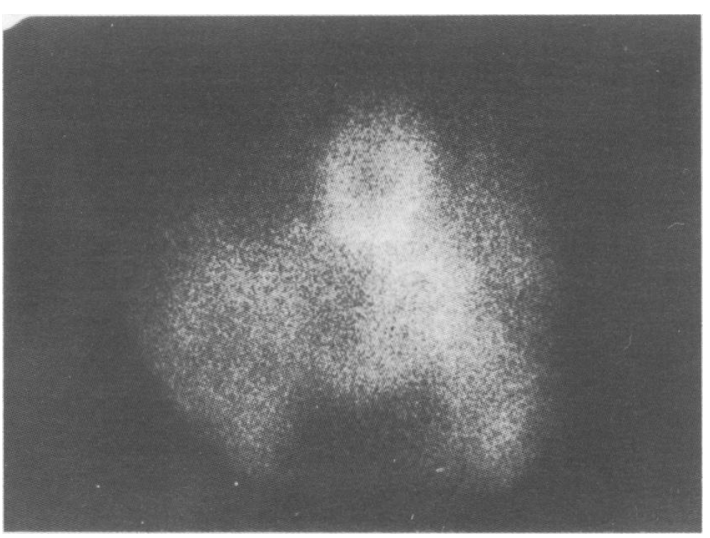

FIG. 2. Thallium 201 perfusion study showing lack of right ventricular uptake (left anterior oblique view).

rub disappeared. Treatment was stopped after seven days and restarted 2 days later because of the recurrence of fever. At this stage, diuretic and saltfree diet therapy were added. Signs of congestive heart failure cleared. The patient continued to do well and was released from hospital in good condition 2 weeks later.

\section{Discussion}

Right ventricular myocardial infarctions can be easily missed (Cohn et al., 1974). A larger number are found at autopsy than are suspected during life (Wartman and Hellerstein, 1948; Isner and Roberts, 
1978). That the right ventricle can be the site of severe myocardial necrosis has only recently been recognized (Cohn et al., 1974). Involvement usually coexists with left ventricle infarction but can be isolated (Wartman and Hellerstein, 1974; Isner and Roberts, 1978; Cohn, 1979). The clinical expression of RVMI depends on the degree of pump dysfunction due to the severity of ischaemia and infarction of the left ventricle, the pulmonary vascular resistance, the site and extent of the RVMI, the systolic movement of the septum and function of the tricuspid and mitral valve (Cohn, 1979; Lorell et al., 1979; Wackers et al., 1978). RVMI should be suspected when venous hypertension coexists with clear lung fields (Cohn $e t$ al., 1974; Cohn, 1979). The routine 12 lead-electrocardiography adds little to the diagnosis of RVMI, as in most cases only the T-wave changes in the inferior wall are seen, and for diagnosis of RVMI right chest leads must be added. A high level of clinical suspicion and modern imaging techniques have revealed a greater incidence of RVMI than was previously appreciated (Wackers et al., 1978; Sharpe et al., 1978). In our patient, clinical symptoms and electrocardiographic findings did not lead to an immediate diagnosis of infarction. Only when diuretics were withheld did the venous distension in the neck make right ventricular dysfunction evident. The prolonged fever and subsequent pleuropericarditis remained unexplained until a diagnosis of a recent myocardial infarction was entertained.

MUGA study demonstrated the diminished systolic performance of the right ventricle and thallium201 scintigraphy confirmed the diagnosis of right ventricular infarction, by showing lack of perfusion to the right ventricle. Technetium-99 pyrophosphate studies were not performed because of the long interval from the suspected infarction, and since it was shown to be of limited value in such cases in our laboratory (Zafrir et al., 1982).

Dressler's syndrome is a common complication of myocardial infarction occurring between the third and ninetieth day after the infarction (Dressler, 1959ฏ Dressler and Leavitt, 1960). In our patient, the clinical picture of prolonged fever and signs of pleuropericarditis, which promptly responded to salicylate treatment, together with firm evidence of recent RVMI, confirmed the diagnosis of Dressler' syndrome.

Dressler's syndrome should be considered seri흥 ously for cases of unexplained pleuropericarditis and should prompt a search for undiagnosed infarction especially of the right ventricle which tends to escapen clinical detection.

\section{References}

CoHN, J.N. (1979) Right ventricular infarction revisited. American Journal of Cardiology, 43, 666.

Cohn, J.N., Guiha, N.H., Broder, M.I. \& Limas, C.J. (1974) Righ ventricular infarction. Clinical and hemodynamic features American Journal of Cardiology, 33, 209.

DRESSLER, W. (1959) The post myocardial infarction syndrome. report on forty-four cases. Archives of Internal Medicine, 103, 280

DressleR, W. \& LeAvitT, S.S. (1960) Pericarditis after acuto myocardial infarction. Journal of the American Medical Associa tion, 173, 1225.

ISNER, J.M. \& ROBERTS, W.C. (1978) Right ventricular infarction complicating left ventricular infarction secondary to coronar heart disease. American Journal of Cardiology, 42, 885.

LORE Ll, B., LEINBACH, R.C., POHOST, G.M., GOLD, H.K. \& DDEO SANCTIS, R.W. (1979) Right ventricular infarction. Amerfarfo Journal of Cardiology, 43, 465.

ShaRPE, D.N., Botvinick, E.H., Shames, D.M., Schiller, NG. Massie, B.M., Chatterjee, K. \& Parmley, W.W. (1978) Tृֶ non-invasive diagnosis of right ventricular infarction. Circulation? 57, 483.

WACKERS, F.J.T., LIE, K.I., SOKOLE, E.B., RES, J., VAN DER̆ SCHOOT, J.B. \& DURRER, D. (1978) Prevalence of right ventricug lar involvement in inferior wall infarction assessed with myocar=dial imaging with thallium 201 and technetium-99m pyrophose phate. American Journal of Cardiology, 42, 358.

WARTMAN, W.B. \& Hellerstein, H.K. (1948) The incidence op heart disease in 2,000 consecutive autopsies. Annals of Internat Medicine, 28, 41.

Zafrir, N., Sclerowsky, S., Lubin, E., Hellman, C., Arditi, A음 \& AGMON, J. (1982) Diagnosis of right ventricular infarction b₹ noninvasive methods. Harefua, 102, 1. 